\title{
Complicações atípicas e características clínico-epidemiológicas do COVID-19: uma revisão integrativa
}

\author{
Atypical complications and clinical-epidemiological characteristics of COVID-19: an \\ integrative review \\ Complicaciones atípicas y características clínico-epidemiológicas de COVID-19: una \\ revisión integradora
}

Larissa Mendes do Monte ${ }^{1 *}$, Larissa Alvim Mendes ${ }^{1}$, Rafaela Lima Carmargo ${ }^{1}$, Rúbia Soares de Sousa Gomes ${ }^{1}$, Pedro Henrique Araújo da Silveira' ${ }^{1}$, Mariana Silotti Cabelino Seyfarth ${ }^{1}$, Darlei Montes Cunha ${ }^{1}$, Lusitânia de Paula Ramos Oliveira ${ }^{2}$, Robson da Silveira ${ }^{1}$, Gládma Rejane Ramos Araújo da Silveira ${ }^{1}$.

\begin{abstract}
RESUMO
Objetivo: Compreender através de uma revisão integrativa a respeito das complicações atípicas e características clínico-epidemiológicas sobre o COVID-19. Métodos: Esse estudo trata-se de uma revisão da literatura do tipo integrativa, cujo objetivo é uma abordagem ampla referente às revisões bibliográficas, além da inclusão de estudos experimentais a respeito da COVID-19. Resultados: A disseminação da patologia dá-se principalmente por gotículas, contato direto com fômites, além de outras formas recémdescobertas de transmissão como a fecal oral, principalmente em crianças. Os sinais clínicos principais da doença em questão são: febre, tosse, mialgia, leucopenia, linfopenia, podendo causar também afecções em outros órgãos como o rim. O COVID-19 pode levar a danos alveolares maciços e insuficiência respiratória progressiva e ao óbito. Sendo importante ressaltar o surgimento de um quadro atípico como anosmia, conjuntivite, manifestações cutâneas, alterações renais e dor abdominal. Considerações finais: Por isso, o entendimento dos profissionais da saúde, acerca dos sintomas atípicos e dos aspectos clínicoepidemiológicos dessa patogênese são necessários, a fim de melhorar as estratégias clínicas de atenção e cuidados contra a doença.
\end{abstract}

Palavras-chave: Fisiopatologia, Infecções por coronavírus, Pneumonia.

\begin{abstract}
Objective: Understand through an integrative review regarding atypical complications and clinicalepidemiological characteristics about COVID-19. Methods: This study is a review of the integrative literature, whose objectiveis a broad approach regarding bibliographic reviews, in addition to the inclusion of experimental studies regarding COVID-19. Results: The dissemination of the pathology occurs mainly through droplets, direct contact with fomites, in addition to other recently forms of transmisión such as fecal oral, mainly in children. The main clinical signs of the disease in question are: fever, cough, myalgia, leukopenia, lymphopenia, which can also cause diseases in other organs such as the kidney. COVID-19 can lead to massive alveolar damage and progressive respiratory failure and death. It is important to highlight the arisement of an atypical condition with anosmia, conjunctivitis, skin manifestations, renal changes and abdominal pain. Final considerations: Therefore, the understanding of health professionals about the atypical symptoms, the clinical and epidemiological aspects of this pathogenesis is necessary in order to improve the clinical strategies of care and also care against the disease.
\end{abstract}

Keywords: Pathophysiology, Coronavirus infections, Pneumonia.

\section{RESUMEN}

Objetivo: Comprender através de uma revisión integradora sobre las complicaciones atípicas y las características clínico-epidemiológicas sobre COVID-19. Métodos: Este estudio es una revisión de la

${ }_{1}^{1}$ Centro Universitário (Uni-Facig), Manhuaçu - MG. *E-mail: lala_mendesmonte@hotmail.com

2 Faculdade UNIVIX, Vitória - ES. 
literatura integradora, cuyo objetivo es un enfoque amplio con respecto a las revisiones bibliográficas, además de la inclusión de estudios experimentales con respecto a COVID-19. Resultados: La diseminación de la patología ocurre principalmente a través de gotitas, contacto directo con fómites, además de otras formas de transmisión recientemente descubiertas, como la fecal oral, principalmente en niños. Los principales signos clínicos de la enfermedad en cuestión son: fiebre, tos, mialgia, leucopenia, linfopenia, que también pueden causar enfermedades en otros órganos como el riñón. COVID-19 puede conducir a daño alveolar masivo e insuficiencia respiratoria progresiva y muerte. Es importante resaltar la aparición de una condición atípica como anosmia, conjuntivitis, manifestaciones cutáneas, cambios renales, y dolor abdominal. Consideraciones finales: Por lo tanto, la compresión de la salud sobre los síntomas atípicos y los aspectos clínicos y epidemiológicos de esta patogénesis son necesarios para mejorar las estrategias clínicas de atención y atención contra la enfermedad.

Palabras clave: Fisiopatología, Infecciones por coronavirus, Neumonía.

\section{INTRODUÇÃO}

Em 31 de dezembro de 2019, a Organização Mundial da Saúde (OMS) foi notificada pela presença de uma doença desconhecida que cursava com pneumonia de origem obscura na cidade de Wuhan, província de Hubei na China, que estava infectando pessoas com um nível exponencial e levando a óbito. Em 30 de janeiro de 2020 foi declarada Emergência de Saúde pública Internacional, devido a gravidade e rápida disseminação da Doença do Coronavírus 19 (COVID-19) pelo mundo. Segundo dados de 1 de abril de 2020 no mundo haviam 827.419 casos confirmados, 40.777 óbitos, 206 países/territórios afetados (WHO, 2020).

A COVID-19 é uma doença recém-descoberta e altamente contagiosa causada pelo Coronavírus com evolução para Síndrome Respiratória Aguda Grave 2 (SARS-CoV-2) (SARALADEVI N, et al., 2020). Os coronavírus (CoV) são patógenos causadores de doenças respiratórias, comuns em infecções em animais. São caracterizados por apresentar ácido ribonucleico (RNA) de fita simples como material genético. Tais vírus podem atravessar barreiras interespécies, e causar doenças. Desconhece-se atualmente a gênese exata do SARS-CoV-2, especula-se, porém que possa ser de origem animal (CASCELLA M, et al., 2020).

Essa patologia demonstrou uma infecção de caráter principalmente respiratório, semelhante a uma gripe. Os sinais clínicos mais observados foram: febre, tosse, mialgia, leucopenia, linfopenia, podendo causar também afecções em outros órgãos como o rim. Algumas pessoas são assintomáticas com quadros leve da doença quando manifestado, já em outras incidem a forma grave, principalmente em indivíduos com doenças crônicas prévias e de idade avançada (SARALADEVI N, et al., 2020).

Declarada a pandemia, a OMS (2020) enfatizou a necessidade de isolamento coletivo a fim de retardar a propagação do COVID-19, incentivando os países a implementar medidas de emergência como a preparação de hospitais e profissionais, a fim de proteger o maior número de pessoas, pois não há tratamento antiviral especifico recomendado, e não existe vacina disponível (WHO, 2020). Desse modo, entender a fisiopatologia da doença em suas diversas facetas e manifestações, mesmo que ainda pouco conhecidas, torna-se primordial para controle e diminuição da morbimortalidade (CASCELLA M, et al., 2020).

Considerando a complexidade da patologia em questão, esse estudo justifica-se pela necessidade de instituir orientações para os profissionais da saúde, uma vez que há escassez de dados sobre a temática. Também, objetiva-se explanar as formas atípicas e características clínico-epidemiológicas do COVID-19 da COVID-19 através de um estudo integrativo e abrangente, proporcionando maior entendimento das manifestações clínicas da doença e disponibilizando instrumentos para embasamento clínico-teórico dos profissionais da saúde.

\section{MÉTODOS}

Trata-se de uma revisão da literatura do tipo integrativa. Para a realização desta pesquisa procedeu-se as seguintes etapas: Elaborada da pergunta "Quais são as complicações atípicas presentes nos casos de COVID-19?"; busca diversificada de artigos, com leitura de resumos e títulos, e posteriormente leitura 
completa; extração de dados com análise crítica de cada estudo e das características; comparação dos resultados entre vários países; e por fim, apresentação da revisão em si.

O levantamento bibliográfico foi feito a partir de trabalhos publicados em periódicos como revistas, jornais e Medical Literature Analysis and Retrieval System Online (MEDLINE), no ano de 2020. Utilizaram-se os Descritores em Ciências da Saúde (DeCS): Coronavírus e Infecções por Coronavírus. Os trabalhos selecionados passaram por critérios de exclusão e inclusão (Figura 1).

Figura 1 - Fluxograma da aplicação dos critérios de inclusão e exclusão.

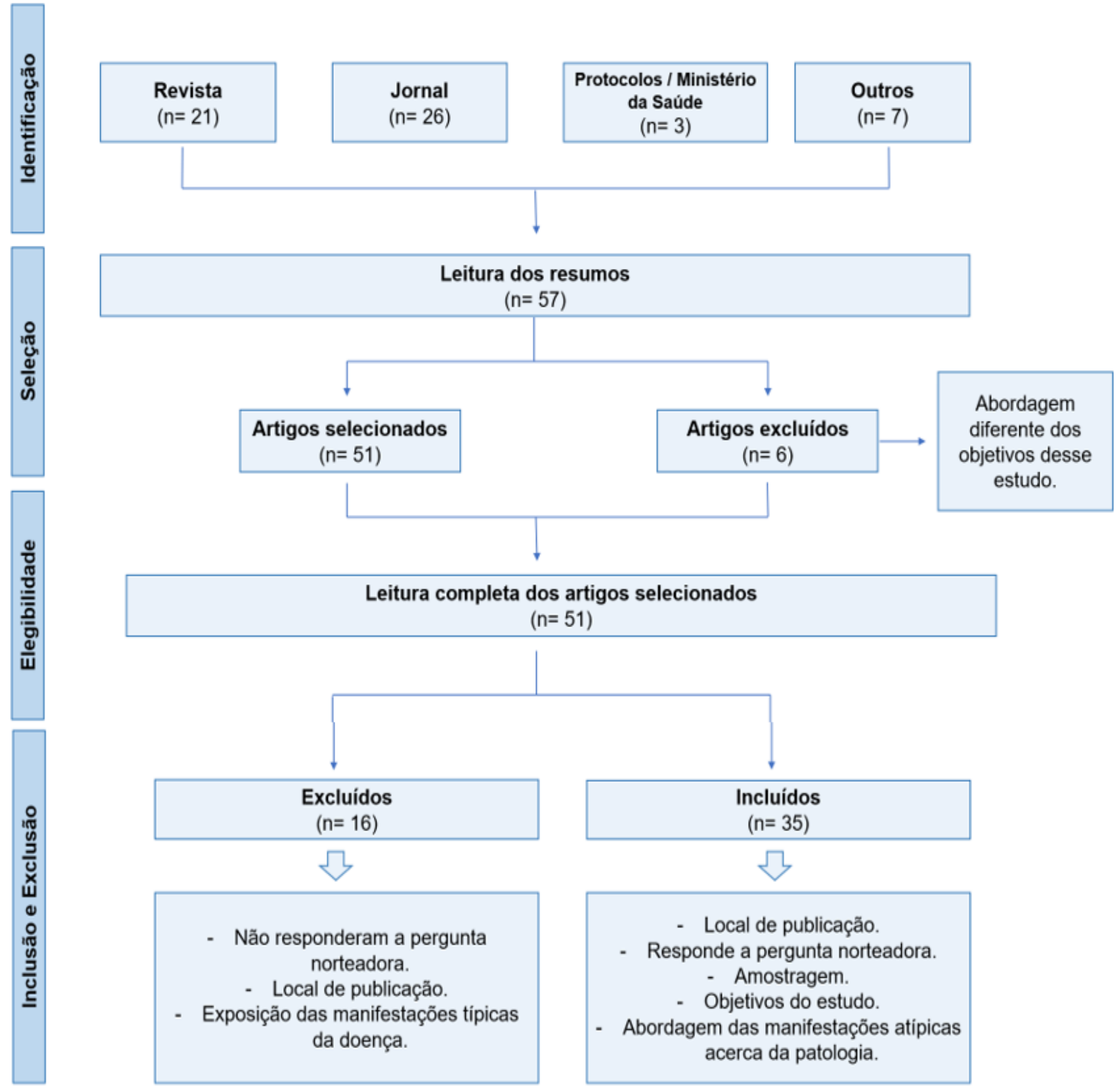

Fonte: Monte LM, et al., 2020.

\section{RESULTADOS}

A partir da aplicabilidade dos critérios de inclusão e exclusão, os resultados presentes neste trabalho abordam os principais achados dos trinta e cinco estudos selecionados que estão expostos a seguir (Quadro 1). 


\section{Revista Eletrônica Acervo Saúde / Electronic Journal Collection Health | ISSN 2178-2091}

Quadro 1 - Estudos selecionados para a pesquisa.

\begin{tabular}{|c|c|c|c|}
\hline Autor/Ano & Periódico & Idioma & Objetivos \\
\hline $\begin{array}{l}\text { Joob BB e Wiwanitkit VV, } \\
\qquad(2020)\end{array}$ & $\begin{array}{l}\text { Journal of the American Academy of } \\
\text { Dermatology }\end{array}$ & Inglês & $\begin{array}{l}\text { Responder uma carta ao Editor, sobre um artigo que relata o uso do } \\
\text { departamento de Dermatologia para atender os pacientes doentes com o } \\
\text { COVID-19. }\end{array}$ \\
\hline Recalti SS (2020). & $\begin{array}{l}\text { Journal of The European Academy of } \\
\text { Dermatology and Venereology }\end{array}$ & Inglês & $\begin{array}{l}\text { Relatar a presença de manifestações cutâneas em } 18 \text { pacientes com } \\
\text { COVID-19, que cursaram com um "Rash". }\end{array}$ \\
\hline Shubiao LS, et al. (2020). & Journal Medic of Virology & Inglês & $\begin{array}{l}\text { Demonstrar três membros da mesma família contaminados com COVID-19 } \\
\text { e suas diferentes manifestações. }\end{array}$ \\
\hline Ansari $P$, et al. (2017). & Manual MSD & Português & Faz uma abordagem a respeito de dor abdominal aguda. \\
\hline Zhialiand Hu, et al. (2020). & Science China Life Sciences & Inglês & $\begin{array}{l}\text { Padrões de TC em pacientes contaminados pelo COVID-19, porém, } \\
\text { assintomáticos. }\end{array}$ \\
\hline $\begin{array}{l}\text { Gao Wenjing e Li Liming } \\
\qquad(2020) \text {. }\end{array}$ & Chinese Journal of Epidemiology & Inglês & $\begin{array}{l}\text { Definir infecção assintomática e alterações bioquímicas detectadas } \\
\text { evidenciadas somente em exames laboratoriais. }\end{array}$ \\
\hline Ganyani TG, et al. (2020). & MedRxiv & Inglês & Demonstrar estudos com transmissão em massa por assintomáticos. \\
\hline Kenneth M (2020). & UpToDate & Inglês & $\begin{array}{l}\text { Relatar as manifestações típicas do COVID-19, além de relatos de locais } \\
\text { que foram encontrados pacientes contaminados, mas assintomáticos. }\end{array}$ \\
\hline Pan L, et al. (2020). & $\begin{array}{l}\text { American Journal of } \\
\text { Gastroenterology }\end{array}$ & Inglês & $\begin{array}{l}\text { Analisar as características clínico-epidemiológicas, focando nos sintomas } \\
\text { gastrointestinais. }\end{array}$ \\
\hline Wu Y, et al. (2020). & $\begin{array}{l}\text { The Lancet Gastroenterology e } \\
\text { Hepatology }\end{array}$ & Inglês & $\begin{array}{l}\text { Analisar a presença prolongada do vírus SARS-CoV-2 em amostras fecais } \\
\text { desde o primeiro dia do surgimento do sintoma. }\end{array}$ \\
\hline Schwartz DA (2020). & $\begin{array}{l}\text { Archives of Pathology \& Laboratory } \\
\text { Medicine }\end{array}$ & Inglês & $\begin{array}{l}\text { Observar os efeitos da doença respiratória transmitida pelo coronavírus, a } \\
\text { SARS-CoV-2, no período de gestação. }\end{array}$ \\
\hline Díaz CA, et al. (2020). & Asociación Española de Pediatria & Espanhol & infecção neonatal por SARS-CoV-2, Espanha. \\
\hline
\end{tabular}

REAS/EJCH | Vol.Esp.46 | e3699 | DOI: https://doi.org/10.25248/reas.e3699.2020 Página 4 de 12 


\section{Revista Eletrônica Acervo Saúde / Electronic Journal Collection Health | ISSN 2178-2091}

\begin{tabular}{|c|c|c|c|}
\hline Autor/Ano & Periódico & Idioma & Objetivos \\
\hline Zhen L, et al. (2020). & MedRxiv & Inglês & $\begin{array}{l}\text { Relatar o cuidado nas disfunções renais de pacientes com COVID-19, } \\
\text { evidenciando a fisiopatologia da doença, quando em acometimento renal. }\end{array}$ \\
\hline Letko M, et al. (2020). & Nature Microbiology & Inglês & $\begin{array}{l}\text { Como os SARS-CoV-2 interagem com a célula dos hospedeiros, sua } \\
\text { ligação com receptores celular e a capacidade de infecção de diferentes } \\
\text { espécies. }\end{array}$ \\
\hline Saraladevi N, et al. (2020). & Kidney International & Inglês & Evidenciar o envolvimento renal na COVID-19. \\
\hline Yufang S, et al. (2020). & Cell Death \& Differentiation & Inglês & $\begin{array}{l}\text { Descrever aspectos clínicos de apresentação do COVID-19 de acordo com } \\
\text { as agressões imunológicas e órgãos acometidos. }\end{array}$ \\
\hline Henry BM e Lippi G (2020). & International Urologyand Nephrology & Inglês & $\begin{array}{l}\text { Realização de meta-análise para confirmação da relação do SARS-CoV-2 } \\
\text { e gravidade de lesão renal crônica. }\end{array}$ \\
\hline Fu D, et al, (2020). & Nephron & Inglês & $\begin{array}{l}\text { Relatar um caso de um homem de } 75 \text { anos infectado com COVID-19 com } \\
\text { doença renal terminal. }\end{array}$ \\
\hline Zhang T, et al. (2020) & Journal of Medical Virology & Inglês & Relatar 3 casos de SARS-CoV-2, em crianças infectadas em Tianjin, China. \\
\hline Brodin P (2020). & Editorial & Inglês & $\begin{array}{l}\text { Compreender o quadro clínico em crianças e as diversas explicações para } \\
\text { esse fenômeno. }\end{array}$ \\
\hline KCDC (2020). & $\begin{array}{l}\text { Korean Center for Disease and } \\
\text { Control and Prevention }\end{array}$ & Inglês & $\begin{array}{l}\text { Evidenciar que na pulação pediátrica, até março de } 2020,(6,3 \%) \text { dos casos } \\
\text { de COVID-19 eram provenientes de crianças com menos de } 19 \text { anos. }\end{array}$ \\
\hline CDC (2020). & $\begin{array}{l}\text { Chinese Center For Disease Control } \\
\text { And Prevention }\end{array}$ & Inglês & $\begin{array}{l}\text { Revisão de } 72.314 \text { casos, evidenciando que em menos de } 1 \% \text { dos casos } \\
\text { de COVID-19 eram crianças com idade inferior a } 10 \text { anos. }\end{array}$ \\
\hline Cascella M, et al. (2020). & Stat Pearls Publishing & Inglês & $\begin{array}{l}\text { Coletar evidências e informações científicas, bem como uma visão geral da } \\
\text { fisiopatologia e história natural da COVID-19. }\end{array}$ \\
\hline
\end{tabular}

REAS/EJCH | Vol.Esp.46 | e3699 | DOI: https://doi.org/10.25248/reas.e3699.2020 Página 5 de 12 


\section{Revista Eletrônica Acervo Saúde / Electronic Journal Collection Health | ISSN 2178-2091}

\begin{tabular}{|c|c|c|c|}
\hline Autor/Ano & Periódico & Idioma & Objetivos \\
\hline WHO (2020). & Organização Mundial da Saúde & Inglês & $\begin{array}{l}\text { Dados epidemiológicos de morbimortalidade e países afetados pelo } \\
\text { COVID-19. }\end{array}$ \\
\hline Bagheri SHR (2020). & MedRxIV & Inglês & Correlacionar sintomas de anosmia e hiposmia ao COVID-19. \\
\hline lacobucci G (2020). & BMJ 2020 & Inglês & $\begin{array}{l}\text { Examinar quão comum pode ser para as pessoas infectadas com COVID } \\
19, \text { sentirem uma perda do olfato (anosmia) ou sabor (ageusia). }\end{array}$ \\
\hline Lewis R (2020). & Medscape & Português & $\begin{array}{l}\text { Relatar os principais sintomas gastrointestinais que podem surgir no início } \\
\text { da COVID-19, podendo ocasionar transmissão fecal-oral. }\end{array}$ \\
\hline Chen L, et al. (2020). & medRxIV & Inglês & $\begin{array}{l}\text { Investigar as manifestações oculares e as características clínicas dos } \\
\text { pacientes com COVID-19. }\end{array}$ \\
\hline Parry NM (2020). & $\begin{array}{c}\text { Medscape's Coronavirus Resource } \\
\text { Center. }\end{array}$ & Inglês & $\begin{array}{l}\text { Destacar os principais passos que os médicos devem tomar para rastrear } \\
\text { pacientes com SARS-CoV-2, proteger-se contra infecções enquanto } \\
\text { cuidam desses pacientes e minimizar a disseminação do vírus no } \\
\text { consultório. }\end{array}$ \\
\hline WHO (2020). & Organização Mundial da Saúde & Inglês & Alerta aos Dermatologistas para manifestações cutâneas do COVID-19 \\
\hline Shaman JS, et al. (2020). & Science & Inglês & $\begin{array}{l}\text { Relatar sobre a porcentagem de doentes assintomáticos e como esses } \\
\text { afetam a transmissibilidade da doença. }\end{array}$ \\
\hline $\begin{array}{l}\text { Sociedade Brasileira de } \\
\text { Dermatologia (2020). }\end{array}$ & $\begin{array}{l}\text { Sociedade Brasileira de } \\
\text { Dermatologia }\end{array}$ & Português & $\begin{array}{l}\text { A SBD pede calma no enfrentamento da doença e faz recomendações aos } \\
\text { dermatologistas em situações de atendimento. }\end{array}$ \\
\hline Ministério da Saúde (2020). & $\begin{array}{l}\text { Biblioteca Virtual em Saúde } \\
\text { do Ministério da Saúde }\end{array}$ & Português & $\begin{array}{l}\text { Por meio do Protocolo de Manejo Clínico para o Novo Coronavírus(2019- } \\
\text { nCoV), visa informar as características gerais a respeito da COVID-19. }\end{array}$ \\
\hline WHO (2020). & Organização Mundial da Saúde & Inglês & OMS quer testes e isolamento de casos suspeitos para conter Covid-19. \\
\hline WHO (2020). & Organização Mundial da Saúde & Inglês & Testes Laboratoriais em suspeitos humanos do covid-19. \\
\hline
\end{tabular}

Fonte: Monte LM, et al., 2020. 


\section{DISCUSSÃO}

O novo COVID-19 surgiu na cidade de Wuhan na China em 2019, e rapidamente se espalhou em todas as províncias, no mês de março de 2020 já estava em outros 58 países. Como ainda existe muita incerteza em relação à transmissibilidade do vírus, as medidas de contenção deste patógeno estão em andamento e a eficácia do distanciamento social sendo testada, além disso, as pesquisas buscam um fármaco capaz de combater o vírus ou uma vacina que seja eficaz para oferecer a imunidade ao COVID 19 e que não tenha danos colaterais graves (SHAMAN JS, et al., 2020).

A COVID-19, por se tratar de uma infecção viral com alto poder de transmissibilidade e apresentar potencial poder de virulência, a OMS (2020), classificou-a como uma Emergência de Saúde Pública de Importância Internacional. Com base nisso, uma análise feita nas variadas publicações e estudos obtidos por meio das bases de pesquisas, confirmou que o COVID-19 pode cursar com quadros assintomáticos e com manifestações atípicas como anosmia, conjuntivite, manifestações cutâneas, alterações renais e dor abdominal. Além disso, alguns estudos relataram o curso da infecção viral pelo COVID-19 durante a gestação e na população pediátrica. Diante disso, é importante que todos os profissionais de saúde aliados aos órgãos governamentais, tenham conhecimento das formas atípicas de apresentação da doença, para que possam elaborar estratégias de reconhecimento e abordagem desses pacientes, a fim de evitar a transmissão do vírus.

\section{Quadro assintomático}

Apresenta curso clínico com sintomas leves, ou mesmo com sintomatologia ausente, mas se transmitido ao grupo de risco poderá levar a óbito. Essa descoberta explica a rápida disseminação geográfica do COVID19 e demonstra que o controle poderá ser difícil. Segundo os dados registrados em Wuhan, os portadores assintomáticos foram $86 \%$ dos casos, sendo diretamente responsáveis pela explosão da epidemia na China (SHAMAN JS, et al., 2020). A infecção assintomática ou também conhecida como infecção subclínica está relacionada à invasão de patógenos no organismo, desencadeando uma resposta imune específica, causando ou não danos teciduais menores, portanto clinicamente não apresenta sintomas, sinais ou mesmo alterações bioquímicas, sendo detectada apenas através de inspeção laboratorial. Além disso, tanto os pacientes no período de incubação quanto às pessoas com infecção assintomática podem disseminar o vírus e tornar-se a fonte da infecção. O que dificulta a identificação efetiva desses portadores assintomáticos, formando brechas nas medidas de prevenção e controle, o que tem um impacto epidemiológico muito importante (WENJING GW e LIMING LL, 2020). Durante um evento da empresa Biogen, nos Estados Unidos ocorreu uma transmissão em massa do COVID-19, pois após o encontro três funcionários que no momento do evento eram assintomáticos testaram positivo para a doença. Mais tarde, 82 pessoas que estavam no mesmo local e tiveram contato foram infectadas. Outro estudo que analisou os casos de Cingapura relata que mais de $50 \%$ das pessoas foram infectados por assintomáticos (GANYANI TG, et al., 2020). Apesar de existirem indivíduos assintomáticos, estes, podem apresentar imagens típicas de Tomografia Computadorizada de Tórax (TC) em vidro fosco e listras nos pulmões e o diagnóstico é feito pela coleta de swab da faringe que identifica o material genético do CODIV-19 (ZHIALIAND HZ, et al., 2020).

A OMS (2020) recomenda que toda a população que se encaixe nos critérios de técnicos de classificação, que são: contato próximo domiciliar com caso confirmado laboratorialmente mais a presença de febre e/ou sintomas respiratórios, no intervalo de 14 dias após o último contato, sejam testadas para o COVID-19. Porém, no momento o Brasil e outros países não dispõem de kits suficientes para realizar uma política abrangente de examinação, e se de fato os casos assintomáticos forem responsáveis pelo crescente número de contaminados a estratégia ideal seria o isolamento social como melhor opção de prevenção do que a realização dos testes de forma disseminada (GANYANI TG, et al., 2020).

\section{Anosmia e COVID-19}

Conforme lacobucci G (2020) existem evidências que em casos anteriores de coronavírus, uma média de 10 a $15 \%$ dos pacientes queixavam-se de perda de olfato após a contaminação pelo vírus. Notou-se que nas regiões onde os testes para COVID-19 foram extensos, como no Reino Unido, e na Coréia do Sul, 30\% desses 
pacientes com testes positivos tiveram anosmia como principal sintoma. Segundo Bagheri SHR, et al. (2020) houve um aumento de pacientes encaminhados para clínica de otorrinolaringológicas no Irã para avaliação de anosmia concomitante a epidemia de COVID-19. Assim, conclui-se que o Coronavírus pode causar danos aos receptores olfativos.

\section{Conjuntivite}

Segundo a Academia Americana de Oftalmologia (2020), o vírus pode ocasionar a conjuntivite, sendo transmitido por contato com aerossol da conjuntiva. Na China nove pacientes hospitalizados com Covid-19 tiveram conjuntivite, estes tinham SARS-CoV-2 nas secreções oculares. Desse modo, podemos concluir que o vírus infecta a conjuntiva e leva a conjuntivite (PARRY NM, et al., 2020). Conforme Chen L, et al. (2020) a duração média da congestão conjuntival foi de 4,9 $\pm 2,6$ dias (média) variando de 2 a 10 dias. $O$ olho seco $(112,20,97 \%)$, a visão turva $(68,12,73 \%)$ e a sensação de corpo estranho $(63,11,80 \%)$ foram classificados como os três principais sintomas oculares relacionados ao COVID-19.

\section{Manifestações Cutâneas}

A pele é um órgão que pode manifestar sinais de diversas patologias internas, como disfunções metabólicas, neoplasias, disfunções nutricionais, reações adversas a medicamentos e, além disso, doenças infecciosas sistêmicas como a dos países tropicais: Dengue, Zika, Chikungunya, Febre Amarela, Sarampo, Escarlatina, Chagas, Leishmaniose, Micoses entre outras que são endêmicas no chamado "Cinturão Tropical" (JOOB BB e WIWANITKIT VV, 2020).

Um relato feito por Joob BJ e Wiwanitki VW (2020) e publicado recentemente no Journal of the American Academy of Dermatology, relata o caso de 1 paciente entre os 48 casos registrados na Tailândia, que apresentou uma erupção cutânea ("rash") do tipo purpúrico com petéquias e com plaquetopenia associada. Os autores negaram a presença de sinais e sintomas respiratórios e o diagnóstico inicial foi dengue. Posteriormente o paciente evoluiu com um quadro respiratório e o COVID-19 foi confirmado através do exame Proteína C Reativa (PCR). Porém, não foram registradas fotografias e nem colhida à biópsia de pele.

No dia 28/03/2020 a Sociedade Brasileira de Dermatologia publicou um texto onde critica o artigo dizendo que não seria possível usá-lo como fonte de informação científica, visto que a falta de exames comprobatórios como o histopatológico que demostraria a extensão da lesão cutânea, sua distribuição anatômica e sua evolução temporal, além de não haver registro fotográfico. Ressalta também, que dentre dos mais de 600.000 casos notificados atualmente, que nenhum apresentou achados similares à deste paciente (SOCIEDADE BRASILEIRA DE DERMATOLOGIA, 2020).

Uma carta ao editor publicada em 26 de março pelo Journal of the European Academy of Dermatology, relata que no Hospital Alessandro Manzoni, em Locco na Itália, foram coletados dados de 88 pacientes internados por COVID-19 sendo que 18 deles desenvolveram manifestações na pele, sendo que 8 cursaram com o início da doença e 10 após a hospitalização. Foi observado a presença de um "rash" de caráter eritematoso em 14 pacientes confirmados, urticária disseminada em 3 e vesículas similares a varicela em 1. Além disso, houve prurido de caráter leve ou ausente, com distribuição na região do tronco e com remissão em poucos dias, não correlacionando com a gravidade da doença. Recalti SR (2020) finaliza a carta concluindo que se pode "especular" que as manifestações cutâneas podem estar ligadas as que ocorrem em infecções virais comuns. Em um hospital na província de Hubei na China foi relatado 3 membros da mesma família diagnosticados com COVID-19 sendo que uma paciente (A) em 4 de Fevereiro de 2020 desenvolveu um eritema disseminado que foi tratado como crise de Urticária, a mesma retorna no dia 08 de Fevereiro com "rash" pequeno localizado nos membros. Os outros 2 membros da família (B e C) não tiveram manifestações dermatológicas, sendo que na Tomografia Computadorizada os pacientes A e B obtiveram um padrão sugestivo de vidro fosco (SHUBIAO LS, et al., 2020).

A recomendação da OMS (2020) é que os dermatologistas estejam em alerta e vigilantes a qualquer erupção cutânea e seus diagnósticos diferenciais, orientando medidas profiláticas adequadas. Pois, não existem dados epidemiológicos que correlacionem manifestações cutâneas com a atual pandemia pelo COVID-19 (ORGANIZAÇÃO MUNDIAL DA SAÚDE, 2020). 


\section{Alterações renais}

As disfunções renais revelam-se como fator de risco para mortalidade. Inicialmente surgem como alterações leves com posterior progressão para deterioração. A lesão renal aguda em pacientes com COVID19 evolui para prognóstico negativo, diferindo de outros prognósticos da doença passiveis de intervenções curáveis (SARALADEVI N, et al., 2020).

Estudos atuais demonstraram que o SARS-CoV-2 utiliza a Enzima Conversora de Angiotensina 2 (ECA 2), como receptor para entrar na célula. Esta não foi expressa exclusivamente em órgãos do sistema respiratório, de forma que apresentou expressões nos órgãos do trato gastrointestinal, e urinários (rim) quase cem vezes maiores que nos pulmões (LETKO M, et al., 2020; ZHEN LI, et al., 2020; YUFANG S, et al., 2020).

Constatou-se que o rim possui um maior número de receptores reninas de ECA 2 que os pulmões, o que leva ao SARS-CoV-2 a lesionar as células tubulares renais agudamente. Este fato está intimamente relacionado a aceleração e a progressão da inflamação iniciado no sistema respiratório. Tais discrasia podem culminar em amplificação das citocinas inflamatórias de forma irreversível induzindo a falência de múltiplos órgãos e inevitavelmente ao óbito (ZHEN LI, et al., 2020).

A lesão renal é evidenciada nos achados de albuminúria maciça, proteinúria, uremia, hematúria, creatinina sérica elevada (ZHEN LI, et al., 2020; SARALADEVI N, et al., 2020).Pacientes com Lesão Renal Crônica (LRC) apresentam grande risco com a infecção pelo patógeno devido supressão imunológica, obtendo pior prognóstico e evoluindo para falência múltipla de órgãos (FU D, et al., 2020). A lesão renal pode ser aventada como fator de risco na COVID-19 (HENRY BM e LIPPI G, 2020).

Esses pacientes estão sujeitos a infecções oportunistas, o que propicia uma maior variação de infecciosidade e de sintomas principalmente naqueles que necessitam de diálise (SARALADEVI N, et al., 2020). O mecanismo exato do envolvimento renal ainda não é totalmente esclarecido, bem como o tratamento da doença, porém, pode ser observado que o acometimento renal é um potencializador de gravidade nos acometidos por COVID-19 devido a presença da ECA 2 (SARALADEVI N, et al., 2020).

\section{Dor abdominal e abdome agudo}

Dentre os diversos sintomas apresentados pelo surto da COVID-2019, o quadro clínico com sintomas digestivos também é comum. A etiologia revelada até o momento refere-se ao dano indireto causado pela resposta inflamatória sistêmica do indivíduo, dano direto aos enterócitos, alteração da microbiota intestinal e do sistema imunológico das mucosas. A apresentação clínica de dor abdominal pode ser sugestiva de inúmeras patologias de caráter inflamatório, obstrutivo, perfurativo, vascular ou hemorrágico. Devido a essa complexidade, esses pacientes são diagnosticados mais tardiamente pelo COVID-19, e consequentemente, seu prognóstico é pior (MINISTÉRIO DA SAÚDE, 2020; ANSARI, 2017).

O estudo realizado por Pan L, et al. (2020), analisou as características clínico-epidemiológicas de 204 pacientes internados em três hospitais de Hubei na China. Dentre os pacientes em estudo, 48,5\% apresentaram uma ou mais queixas gastrointestinais. Sendo importante salientar-se que, 92 desenvolveram sintomas respiratórios associados e 7 pacientes não queixaram de sintomas respiratórios, tendo somente queixas gastrointestinais como, anorexia, diarreia, vômito e dor abdominal. Aqueles pacientes com manifestações respiratórias demoraram em torno de 7 dias do início dos sintomas até admissão hospitalar, já os pacientes com queixas gastrointestinais demoram 9 dias. Em consequência disso, a taxa de cura foi maior entre os pacientes que não apresentaram alterações digestivas.

Segundo Lewis R (2020), nos Estados Unidos, o primeiro paciente com alteração intestinal, testou positivo para COVID-19, e posteriormente foi detectada a presença do vírus nas fezes. De acordo com uma pesquisa feita por Wu Y (2020), utilizando 98 pacientes, cujo objetivo foi analisar a presença prolongada do vírus SARSCoV-2 em amostras fecais desde o primeiro dia do surgimento do sintoma. Inicialmente, foram coletadas amostras respiratórias e fecais de 74 pacientes, dentre essas 33 amostras fecais deram resultado negativo para o vírus RNA, já em relação às amostras respiratórias foram positivas em média de 15,4 dias. No entanto, 41 pacientes obtiveram amostras positivas na realização do exame fecal, permanecendo em média 27,9 dias, 
já as respiratórias ficaram positivas em média de 16,7 dias. Apesar disso, o risco de contrair o vírus através das fezes é baixo, embora haja hipóteses que o vírus esteja presente nas fezes em alguns casos.

\section{Complicações do vírus na gravidez}

Levando em consideração as epidemias anteriores que resultaram em danos as gestantes como, morbimortalidade materna, transmissão materno-fetal e infecção perinatal, evoluindo com óbito, estudos vêm sendo realizados com intuito de analisar os efeitos danosos da COVID-19 em gestantes. O estudo de Schwartz DA (2020), observou os efeitos da doença respiratória transmitida pelo coronavírus, a SARS-CoV2,no período de gestação. Inicialmente, utilizou-se 38 grávidas que testaram positivo para o COVID-19, nessa avaliação incluiu análise dos exames clínicos, laboratoriais, virológicos e o poder de transmissão via intrauterina ou transplacentária das gestantes aos fetos. Foram coletadas amostras neonatais e, posteriormente testadas, dando como resultado negativo por Reação em Cadeia da Polimerase via Transcriptase Reversa (rt-PCR) para SARS-CoV-2. Concluindo-se no estudo que, não há confirmação de que a doença sofra transmissão intrauterina ou transplacentária de gestantes infectadas para seus fetos, necessitando de mais estudo para determinar a comprovação.

No mês de março de 2020, na Espanha, relatou o primeiro caso de infecção neonatal por SARS-CoV-2. A puérpera no terceiro dia de internação, começou a apresentar alterações como febre, e após dois dias desenvolveu picos febris e quadro respiratório, foi realizada radiografia de tórax, evidenciando pneumonia bilateral grave. A paciente negou viagens a áreas de risco ou contato com pessoas infectadas, no entanto, seu parceiro estava com quadro clínico febril e sintomas de gastroenterite. Foi solicitado a realização do exame de diagnóstico por rt-PCR COVID-19 a ambos, dando resultado positivo. Posteriormente, no sexto dia de vida, efetuou o teste no bebê por aspirado nasofaríngeo, obtendo resultado negativo. Já em isolamento, após 36 horas, foi coletado a segunda amostra, dando positividade com quadro assintomático. Diante ao exposto no estudo, suspeitam que o caso se trata de uma transmissão horizontal (DÍAZ CA, et al., 2020).

\section{Doença na criança}

Existem poucas evidências e relatos detalhados sobre COVID-19 em crianças (TONGQIANG Z, et al., 2020). Nessa perspectiva estudos recentes evidenciaram que as crianças se tornam reservatórios virais, sem apresentar sintomas expressivos, podendo disseminar o vírus a outras pessoas (BRODIN P, 2020).

O Korean Center for Disease and Controland Prevention (KCDC) fez um estudo bastante representativo na população pediátrica, evidenciando que até março de 2020, (6,3\%) dos casos de COVID-19 eram crianças com menos de 19 anos (KCDC, 2020). As razões da gênese do quadro ameno nessa faixa etária permanecem obscuras, havendo apenas algumas teorias sobre a patogênese. O sistema imunológico das crianças é diferente dos adultos, que pode levar a propiciar as diferenças clínicas encontradas em crianças acometidas com SARS-CoV-2, como pode ser explicado através de fatores como: existência de anticorpos maternos em recém-nascidos; resposta qualitativamente diferente ao coronavírus que os adultos; presença de outros vírus nas vias aéreas que podem competir com o vírus e restringir sua proliferação; diferença na expressão dos receptores da ECA 2 necessário pra ligação do coronavírus (BRODIN P, 2020).

A disseminação da doença dá-se principalmente por gotículas, contato direto com fômites (TONGQIANG $Z$, et al., 2020). Uma transmissão secundária incomum é pelas fezes. Um estudo realizado em Tianjin, na China, relata três casos de SARS-CoV-2 em crianças, evidenciando a possibilidade da transmissão fecal-oral. Nesses pacientes, foi realizado swab de orofaringe, o qual tronou-se negativo em média de 11 dias após o diagnóstico. Entretanto após a alta todos casos foram testados com SARS-CoV-2 em amostra de fezes, que demonstrou positividade, apesar de negativos em amostras de garganta (TONGQIANG Z, et al., 2020).

No Hospital Infantil Wuhan crianças sintomáticas e assintomáticas com contato com indivíduos suspeitos de infecção por SARS-CoV-2 foram avaliadas através de esfregaço nasofaringe para detecção do material genético do vírus. Das 1391 crianças avaliadas, 171(12,3\%) foram confirmadas com o SARS-CoV-2. Sinais como febre foi encontrado em $41,5 \%$ das crianças, outros achados incluem tosse e eritema faríngeo. Opacidade em vidro fosco bilateral foi achado comum nos investigados (32,3\%). O estudo evidenciou que a 
grande maioria das crianças apresentava o curso da doença de forma mais branda, além de serem na maioria das vezes assintomáticos (TONGQIANG Z, et al., 2020).

Uma pesquisa feita pelo Chinese Center for Disease Controland Prevention (CDC) com 72.314 casos, demonstrou que menos de $1 \%$ dos casos da infecção incidem em crianças com menos de 10 anos, constatando que em geral em vários países as crianças positivas para COVID-19 apresentam quadro leve da doença, quando não são assintomáticas (CDC, 2020).

\section{CONSIDERAÇÕES FINAIS}

Essa revisão integrativa possibilitou o entendimento a respeito do amplo poder de disseminação do COVID-19 que se tornou uma pandemia. Podemos concluir que a maioria de casos evolui tipicamente, porém, estudos demonstraram quadros assintomáticos e pacientes cursando com anosmia, conjuntivite, insuficiência renal e manifestação cutâneas, além de casos da infecção pelo COVID-19 durante a gestação e na população pediátrica. Desse modo, é essencial o desenvolvimento de novas pesquisas, publicação de estudos, para que possamos compreender todas as formas de evolução dessa patologia. Além disso, os resultados obtidos nesse estudo contribuem para a melhoria de assistência aos pacientes com COVID-19, ajudando dessa forma a conter a transmissibilidade da doença e reduzindo assim a morbimortalidade.

\section{REFERÊNCIAS}

1. ANSARI P. Dor abdominal aguda. Manual MSD, 2017.

2. BAGHERI SHR, et al. Coincidence of COVID-19 epidemic and olfactory dysfunction outbreak. MedRxiv, 2020.

3. BRODIN P. Why is COVID-19 so mild in children? Editorial, 2020.

4. CASCELLA M, et al. Features, Evaluation and Treatment Coronavirus (COVID-19). Stat Pearls Publishing. Treasure Island (FL), 2020.

5. CHEN L, et al. Ocular manifestations and clinical characteristics of 534 cases of COVID-19 in China: A cross-sectional study. MedRxiv, 2020.

6. CHINESE CENTER FOR DISEASE CONTROL AND PREVENTION, CDC 2020. In: Vital Surveillances: The Epidemiological Characteristics of an Outbreak of 2019 Novel Coronavirus Diseases (COVID-19) - China, 2020. Disponível em: <http://weekly.chinacdc.cn/en/article/id/e53946e2-c6c4-41e9-9a9b-fea8db1a8f51>. Acesso em 01 abr. 2020.

7. DÍAZ CA, et al. Primer caso de infección neonatal por SARS-CoV-2 en España. Asociación Española de Pediatria, 2020.

8. FU D, et al. COVID-19 Infection in a Patient with End-Stage Kidney Disease. Nephron, 2020

9. GANYANI T, et al. Estimating the generation interval for COVID-19 based on symptom onset data. MedRxiv, 2020.

10. HENRY B e LIPPI G. Chronic kidney disease is associated with severe coronavirus disease 2019 (COVID-19) infection. International Urology and Nephrology, 2020.

11. IACOBUCCI G. Sixty seconds on. anosmia. BMJ 2020; 368.

12. JOOB BB e WIWANITKIT VV. COVID-19 can present a rash be mistaken for Dengue. Journal of the American Academy of Dermatology, 2020.

13. KOREAN CENTER FOR DISEASE AND CONTROL AND PREVENTION, KCDC 2020. In: Press releases. Disponível em: <https://www.cdc.go.kr/board/board.es?mid=a30402000000\&bid=0030>. Acesso em 01 abr. 2020.

14. LETKO M, et al. Functional assessment of cell entry and receptor usage for SARS-CoV-2 and other lineage $B$ betacoronaviruses. Nature Bicrobiology, 2020; 5(4):562-569.

15. LEWIS R. Sintomas gastrointestinais iniciais na Covid-19 podem indicar transmissão fecal-oral. Medscape, 2020.

16. MCINTOSH KK. Coronavirus disease 2019 (COVID-19). UpToDate, 2020.

17. MINISTÉRIO DA SAÚDE. Protocolo de Manejo Clínico para o Novo Coronavírus (2019-nCoV). Ministério da Saúde: Brasília, 2020.

18. ORGANIZAÇÃO MUNDIAL DA SAÚDE. Alerta aos Dermatologistas para manifestações cutâneas do COVID-19. Organização Mundial da Saúde, 2020.

19. ORGANIZAÇÃO MUNDIAL DA SAÚDE. OMS quer testes e isolamento de casos suspeitos para conter Covid-19. Organização Mundial da Saúde, 2020.

20. PAN L, et al. Clinical characteristics of COVID-19 patients with digestive symptoms in Hubei, China: a descriptive, cross sectional, multicenter study. American Journal of Gastroenterology, 2020. 
21. PARRY NM. AAO Releases COVID-19 Updates for Ophthalmologists. 2020. Medscape's Coronavirus Resource Center, 2020.

22. RECALTI SS. Cutaneous manifestations in COVID-19: a first perspective. Journal of The European Academy of Dermatology and Venereology, 2020.

23. SARALADEVI N, et al. The novel coronavirus 2019 epidemic Q1 and kidneys. Kidney International, 2020.

24. SCHWARTZ DA. An Analysis of 38 Pregnant Women with COVID-19, Their Newborn Infants, and Maternal-Fetal Transmission of SARS-CoV-2: Maternal Coronavirus Infections and Pregnancy Outcomes. Archives of Pathology \& Laboratory Medicine, 2020.

25. SHAMAN J, et al. Substantial undocumented infection facilitates the rapid dissemination of novel coronavirus (SARSCoV2). Science, 2020.

26. SHUBIAO LS, et al. Alert for non-respiratory symptoms of Coronavirus Disease 2019 (COVID-19) patients in epidemic period: A case report of familial cluster with three a symptomatic COVID-19 patients. Journal Medic of Virology, 2020.

27. SOCIEDADE BRASILEIRA DE DERMATOLOGIA. Pandemia de Covid-19: SBD pede calma no enfrentamento da doença e faz recomendações aos dermatologistas em situações de atendimento. Sociedade Brasileira de Dermatologia, 2020.

28. WENJING GG e LIMING LL. Advances on pre symptomatic or asymptomatic carrier transmission of covid-19. Chinese Journal of Epidemiology, 2020; 41.

29. WORLD HEALTH ORGANIZATION WHO, 2020. In: Global research on coronavirus disease (COVID-19). Disponível em: https://www.who.int/emergencies/diseases/novel-coronavirus-2019/global-research-on-novelcoronavirus-2019-ncov. Acesso em 01 abr. 2020.

30. WORLD HEALTH ORGANIZATION WHO. Laboratory testing for coronavirusdisease2019 (COVID-19) in suspected human cases. Organização Mundial da Saúde, 2020.

31. WU Y, et al. Prolonged presence of SARS-CoV-2 viral RNA in fecal samples. The Lancet Gastroenterology e Hepatology, 2020.

32. YUFANG S, et al. COVID-19 infection: the perspectives on immune responses. Cell Death \& Differentiation, 2020

33. ZHANG T, et al. Detectable SARS-CoV-2 Viral RNA in Feces of Three Children during Recovery Period of COVID-19 Pneumonia. Journal of Medical Virology, 2020.

34. ZHEN L, et al. Caution on Kidney Dysfunctions of COVID-19 Patients. MedRxiv, 2020.

35. ZHIALIAND $\mathrm{H}$, et al. Clinical characteristics of 24 asymptomatic infection with COVID-19 screened among close contacts in Najing, China. Science China Life Sciences, 2020. 